\title{
Evolutionary Search for Cellular Automata that Exhibit Self-Organizing Properties Induced by External Perturbations
}

\author{
Yusuke Iwase, Reiji Suzuki and Takaya Arita \\ Graduate School of Information Science, Nagoya University \\ Furo-cho, Chikusa-ku, Nagoya 464-8601, Japan \\ E-mail: iwase@alife.cs.is.nagoya-u.ac.jp, \{reiji, arita\}@nagoya-u.jp
}

\begin{abstract}
Cellular Automata (CAs) have been investigated extensively as abstract models of the distributed systems composed of autonomous entities characterized by local interaction. However, how CAs can interact with their external environment still needs discussion. This paper aims at understanding emergent properties of CAs induced by external perturbations. We assumed a task in which a $\mathrm{CA}$ has to change its global state distinguished by the distribution ratio of cell states after every occurrence of a perturbation period. In the perturbation period, each cell state is modified by using an external rule with a small probability. By conducting evolutionary searches for rules of CAs that can solve this task, we obtained interesting behaviors of CAs in which their global state cyclically transited among different stable states in either ascending or descending order. Detailed analyses showed that such behaviors are due to their self-organizing properties that a drastic change in their global state occurs every when the number of the subsequent dominant cell state goes beyond a certain threshold through occurrences of perturbation periods.
\end{abstract}

\section{INTRODUCTION}

A cellular automaton (CA) is a discrete model consisting of a regular grid of finite automata called cells. The next state of each cell is completely decided by the current states of its neighbors including itself. Therefore CAs are wellsuited for investigating the global behavior emerging from local interactions among component parts.

There have been a number of studies on basic characteristic of CAs under the assumption of strong restrictions such as a regular arrangement of cells and synchronous update of the cell states. It is well known that Wolfram suggested that elementary (one-dimensional two-state three-neighbor) CAs fall into four classes. In particular, he pointed out that CAs in class four exhibit complex behaviors, and some of them achieve computational universality[1].

On the one hand, there are studies on CAs with relaxed restrictions so as to investigate the behaviors of the decentralized systems in realistic situations from a variety of view points. Ingerson and Buvel pointed out that the synchronous updating rule is unnatural if we regard them as abstractions of decentralized systems in a real world, and analyzed elementary CAs with asynchronous updating rules[2]. They demonstrated that the global pattern of the asynchronous CA self-organized into a regular array, and argued that asynchronous CA might be useful for understanding selforganization in a real world.

Also, there are several discussions on the effects of influences on the system from outside such as boundary conditions and perturbations. Ninagawa et al. focused on influences of the differences, and compared the effects of the dissipative boundary conditions (in which each cell state is randomly decided at each step) on the global behaviors of CAs with those of the standard periodic boundary conditions[3]. They showed that the CAs with the former condition can eliminate the size effects of the grid on the global behaviors which are prominent in CAs with the latter condition. Marr et al. showed that based on comparison of space-time diagrams of configurations and some of indices, the modifications of the neighboring structures on the regular grid can be functionally equivalent to the introduction of stochastic noises on the states of the cells in CAs that basically exhibit chaotic behaviors[4].

In particular, Roli et al. focused on the influences of the external world on autonomous distributed systems, and constructed asynchronous CA with external perturbations termed "dissipative cellular automata" in that the external environment can somehow inject energy to dynamically influence the evolution of the automata[5][6]. They regarded the asynchronous $\mathrm{CA}$ as a group of autonomous individuals which locally interact with each other, and introduced continuously occurring perturbations on the states of the cells into their model. The perturbations correspond to the influences caused by the external world on the group. The CAs presented regular patterns if and only if they are induced by the perturbations, such as a stripe pattern. Moreover, they argued about applications of such self-organized features for controlling decentralized pervasive computing systems such as sensor networks. For example, based on the experiments of dissipative CAs, they showed possible scenarios that the global state of the CA can be changed to the desired pattern only by filling up the limited area of the CA with a fixed pattern of the states. However, these discussions were based on several hand-coded rules for CAs. Thus, it is still an open question how CAs can show emergent properties through the interactions with an external world.

However, in general, there are complex relationships between the global behaviors of CAs and the local behaviors of cells. It is difficult to design the rules for CAs by hand-coding which exhibit the desired emergent behaviors. Thus, there have been various studies based on evolutionary searches for rules of CAs that can exhibit emergent behaviors[7]. Rocha evolved CA rules that can solve the density task, and discussed about the nature of the memory-like interactions 


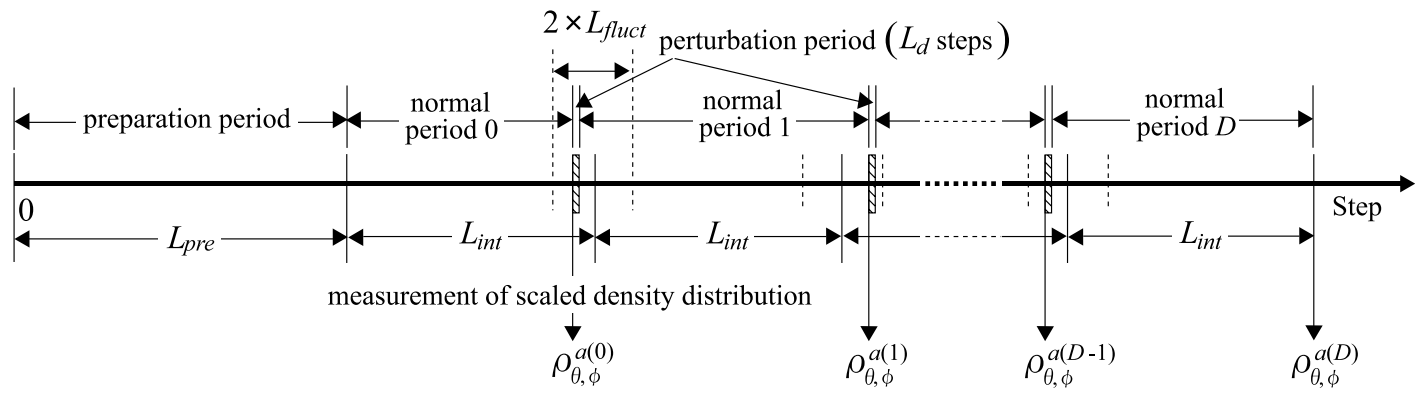

Fig. 1. Repeated occurrences of external perturbations.

among the particles of the cell states emerged for storing and manipulating information[8]. Ninagawa also evolved CAs which generate 1/f noise where the power is inversely proportional to the frequency, and obtained a CA of which behavior is similar to the Game of Life[9]. As above, an evolutionary search for rules of CAs will be also useful for understanding the characteristics of systems that exhibit selforganizing properties caused by interactions with an external environment.

Our purpose is to clarify emergent properties of CAs induced by external perturbations as a first step toward understanding interactions between CAs and external environments. In this paper, we assumed a task in which CAs have to change its global state after every occurrence of perturbation period, and searched the rules for CAs which can solve the task by using a genetic algorithm. We obtained the rules for CAs in which global state of evolved CAs cyclically transited among different stable states of which the number is more than that of distinct cell states, and looked into the self-organizing properties that a drastic change in its global state occurs every two successive occurrences of perturbation periods.

\section{TASK}

We constructed a task which self-organizing behaviors induced by external perturbations are required to solve. In a evaluation process of a CA, there are the fixed number of perturbation periods in which each cell state is modified by using an external rule with a small probability. The CA has to change its configuration represented by the distribution ratio of cell states after every occurrence of a perturbation period. This is non-trivial if the number of the perturbation periods is larger than the number of possible cell states because the global behavior of CAs must stably exhibit different configurations composed of intermediate distribution ratios. Thus the CAs should need some kind of emergent properties that utilize the occurrences of perturbations effectively.

\section{A. Cellular Automata and Perturbations}

We adopt a two-dimensional $(N \times N) M$-state nineneighbor (moore neighborhood) cellular automata with periodic boundary condition as an abstract model of the dis- tributed systems composed of autonomous entities characterized by local interactions.

A cell $(i, j)$ have a state $q_{i, j}^{t} \in\{0, \ldots, M-1\}$ at time step $t$. At each time step $t$, each cell state is asynchronously updated with a probability $P_{a}$ by

$$
q_{i, j}^{t+1}= \begin{cases}\delta\left(S_{i, j}^{t}\right) & \left(P_{a}\right) \\ q_{i, j}^{t} & \left(1-P_{a}\right),\end{cases}
$$

where $\delta$ is a local transition rule which maps a configuration of cell states in a neighborhood $(3 \times 3$ cells around the focal cell $(i, j)) S_{i, j}^{t}$ into a state.

Also we introduce an external perturbation $\epsilon$ which changes a cell state independently of $\delta . \epsilon$ expresses a simple transition rule that increments the value of the cell state as defined by

$$
\epsilon: q_{i, j}^{t+1}:=\left(q_{i, j}^{t+1}+1\right) \bmod M .
$$

$\epsilon$ is applied to each cell with a probability $P_{e}$ every after transitions of cell states by Eq. (1). We expect that some of relationship between the global behavior of CAs and external perturbations can occur by introducing Eq. (2). It is because that the actual effect of a perturbation on the CA (a change in the cell state) is deterministic although it occurs probabilistically.

\section{B. Transition and Evaluation}

Fig. 1 is a time diagram of an evaluation process for a rule $(\delta)$ of a CA described above. Starting from the initial condition in which each cell state is randomly assigned, the transitions without perturbations $\left(P_{e}=0.0\right)$ occur for $L_{\text {pre }}$ steps so that effects of the initial condition are eliminated. Next, a perturbation period of $L_{d}$ steps occurs every after a normal period of approximately $L_{i n t}$ steps. For each cell, a perturbation occurs with a probability $P_{e}=\beta$ during perturbation periods, and it does not occur during normal periods $\left(P_{e}=0\right)$. The evaluation stops when the normal periods have occurred for $D+1$ times. Note that the actual time step at which each perturbation period starts fluctuates randomly within a specific range ( $\pm L_{\text {fluct }}$ steps) as shown in Fig. 1. 


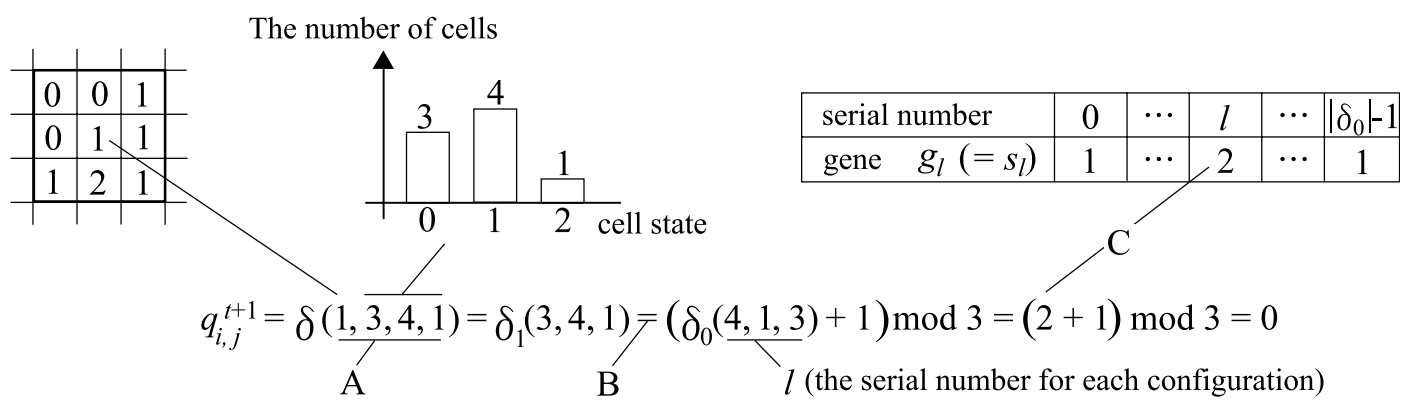

Fig. 2. An example of applying the transition rules $(M=3)$.

A density distribution of cell states $\rho^{t}$ at time $t$ is a vector consisting of the ratios $\rho^{t}(s)$ of the cell state $s$ among all cells, which is defined by

$$
\begin{aligned}
\rho^{t}(s) & =\frac{1}{N \times N} \sum_{(i, j) \in N \times N} \begin{cases}1 & \text { if } q_{i, j}^{t}=s \\
0 & \text { otherwise }\end{cases} \\
\boldsymbol{\rho}^{t} & =\left\{\rho^{t}(0), \rho^{t}(1), \ldots, \rho^{t}(M-1)\right\} .
\end{aligned}
$$

Also, in order to stress the the existence of a small amount of each cell state, we define a scaled density distribution $\boldsymbol{\rho}_{\theta, \phi}^{t}$ by

$$
\begin{aligned}
S F_{\theta, \phi}(x) & =\frac{1}{1+e^{-(x-\theta) \times \phi}}, \\
\rho_{\theta, \phi}^{t}(s) & =S F_{\theta, \phi}\left(\rho^{t}(s)\right), \\
\boldsymbol{\rho}_{\theta, \phi}^{t} & =\left\{\rho_{\theta, \phi}^{t}(0), \rho_{\theta, \phi}^{t}(1), \ldots, \rho_{\theta, \phi}^{t}(M-1)\right\},
\end{aligned}
$$

where $S F_{\theta, \phi}$ is a sigmoid function which scales $\rho^{t}(s)$ with a threshold $\theta$, and $\phi$ is a parameter for a degree of scaling. Eq. (4) means that if $\rho^{t}(s)$ is more than $\theta, \rho_{\theta, \phi}^{t}(s)$ becomes close to 1 . Otherwise, it becomes close to 0 .

Here, we take $\rho_{\theta, \phi}^{t}$ as the global configuration of the CA, and define the fitness by using $\boldsymbol{\rho}_{\theta, \phi}^{t}$ at the last steps of normal periods $\left(\boldsymbol{\rho}_{\theta, \phi}^{a(0)}, \boldsymbol{\rho}_{\theta, \phi}^{a(1)}, \ldots, \boldsymbol{\rho}_{\theta, \phi}^{a(D)}\right.$ in Fig. 1) as follows:

$$
\begin{aligned}
&\left|\boldsymbol{\rho}_{\theta, \phi}^{i}-\boldsymbol{\rho}_{\theta, \phi}^{j}\right|=\left|\rho_{\theta, \phi}^{i}(0)-\rho_{\theta, \phi}^{j}(0)\right|+ \\
&\left|\rho_{\theta, \phi}^{i}(1)-\rho_{\theta, \phi}^{j}(1)\right|+\cdots+ \\
& f_{\theta, \phi}=\left|\rho_{\theta, \phi}^{i}(M-1)-\rho_{\theta, \phi}^{j}(M-1)\right| \\
& \sum_{i \neq j \in\{a(0), a(1), \ldots, a(D)\}}\left|\boldsymbol{\rho}_{\theta, \phi}^{i}-\boldsymbol{\rho}_{\theta, \phi}^{j}\right| .(6)
\end{aligned}
$$

Eq. (5) defines the difference between the scaled density distributions as the sum of the absolute differences between the corresponding elements of the distributions. The fitness of $\delta$ is the sum of the differences over all possible pairs of the scaled density distributions at the last steps of the normal periods.

Thus, the CAs have to use an occurrence of a perturbation period as a trigger to change their own global configuration dynamically.

\section{Evolutionary Search by Genetic Algorithm}

\section{A. Transition Rule}

In this paper, we adopt a transition rule based on the number of each cell state in the neighborhood, expecting emergence of interesting behaviors of the $\mathrm{CA}$ and reduction of the search domain for a genetic algorithm (GA).

Fig. 2 illustrates an example of the transition rules in the case of $M=3$. This rule is an extended version of outertotalistic rules. The pattern of the neighborhood configuration $S_{i, j}^{t}$ at the cell $(i, j)$ is given by

$$
\begin{aligned}
n_{i, j}^{t}(s) & =\sum_{|k| \leq 1,|l| \leq 1,|k|+|l| \neq 0}\left\{\begin{array}{l}
1 \text { if } q_{i+k, j+l}^{t}=s \\
0 \text { otherwise },
\end{array}\right. \\
S_{i, j}^{t} & =\left\{q_{i, j}^{t}, n_{i, j}^{t}(0), n_{i, j}^{t}(1), \ldots, n_{i, j}^{t}(M-1)\right\} .
\end{aligned}
$$

$n_{i, j}^{t}(s)$ is the number of the cell state $s$ in the neighborhood except for the cell $(i, j)$ itself. $S_{i, j}^{t}$ is a set of the focal cell state and the number of each cell state as illustrated in Fig. 2 - A.

We further introduced a transitivity into the transition rule $\delta$ defined as follows:

$$
\begin{gathered}
\delta_{q}(n(0), n(1), \ldots, n(M-1)) \\
=\delta(q, n(0), n(1), \ldots, n(M-1)), \\
\delta_{q}(n(0), n(1), \ldots, n(M-1)) \\
=\quad\left(\delta_{0}(n(\operatorname{cs}(q, 0)), n(\operatorname{cs}(q, 1)), \ldots,\right. \\
n(c s(q, M-1)))+q) \bmod M \\
(c s(q, x)=(q+x) \bmod M),
\end{gathered}
$$

where $\delta_{q}$ is a map from a neighboring configuration in which the focal cell state is $q$ to a state. Eq. (8) means that $\delta_{q}$ $(q=1, \ldots, M-1)$ can be obtained by incrementing each value in the equation of $\delta_{0}$ as shown in Fig. 2 - B.

The above description of the rule largely reduces the search domain for GA. In case of employing twodimensional $M$-state nine-neighbor CAs, the maximum number of the transition rules is $M^{M^{9}}$ because there are $M^{9}$ distinct neighborhood configurations at a maximum. On the contrary, if we adopt rules described above, the possible 
number comes down to $M^{8+(M-1)} C_{M-1} 1$.

\section{B. Genetic Information and Evolutionary Operations}

We used a form of GA to evolve CAs to perform the task described above. Each individual in GA has a string consists of genes $g_{l}$. Each $g_{l}$ represents the cell state into which the corresponding pattern of the neighborhood is mapped in $\delta_{0}$ (Fig. 2 - C). The population of $I$ individuals is evolved over $G$ generations as follows:

1) All $g_{l} \mathrm{~s}$ in the initial population is initialized with 0 .

2) The string of genes of each individuals is translated to the transition rule, and then its fitness is calculated by using the evaluation process described above.

3) The top $E$ individuals are copied without modification to the next generation. The remaining $I-E$ individuals for the next generation are selected with the probability equal to its relative fitness within the whole population, and they pair with each other as parents.

4) Two offsprings are generated from the pair based on a two-point crossover with a probability $P_{\text {crossover }}$ and a mutation for each gene with a probability $P_{\text {mutation }}$. A mutation changes $g_{l}$ to a random value $\left(0 \leq g_{l}<M\right)$ except for the current value.

5) The $E$ elites and $I-E$ offsprings form the population of the new generation, and the process goes back to the step 2 until the generation reaches $G$.

\section{EXPERIMENTAL RESUlts AND ANALYSES}

\section{A. Course of Evolution}

We adopted the settings of parameters as follows: $N=64$, $M=3, P_{a}=0.2, \beta=0.01, D=5, L_{\text {pre }}=2048, L_{\text {int }}=$ $1024, L_{\text {fluct }}=128, L_{d}=8, \theta=0.1, \phi=100, I=32$, $E=8, G=256, P_{\text {crossover }}=0.75$ and $P_{\text {mutation }}=0.05$.

We conducted 12 runs, and it turned out that the fitness reached approximately 27 in 10 runs. The fitness of remaining 2 runs went up to about 21 or 25 . Here, we focus on the run in which the fitness reached the best value among them.

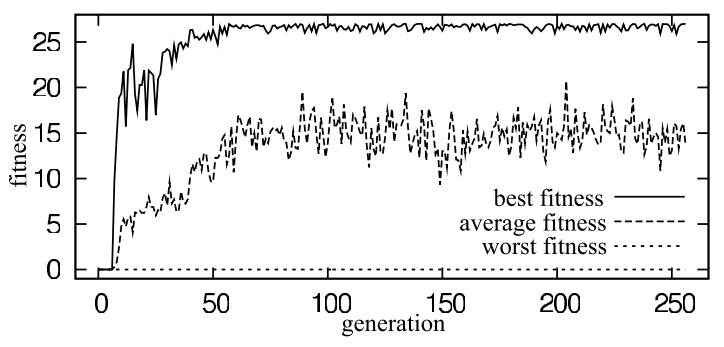

Fig. 3. The fitness of the population.

Fig. 3 shows the best, average and worst fitness at each generation. The best fitness was about zero in the initial population and it rapidly went up to approximately 19 until the

\footnotetext{
${ }^{1}$ In the case of $M=3$, the every possible number of the transition rule in our model comes down from $M^{M^{9}}=3^{3^{9}} \approx 1.505 \times 10^{9391}$ to $M^{8+(M-1)} C_{M-1}=3^{10} C_{2} \approx 2.954 \times 10^{21}$.
}

10th generation, then eventually converged to approximately 27 around the 50th generation. Also, we see the average fitness tended to be a half of the best fitness, and the worst fitness was almost zero through the experiment.

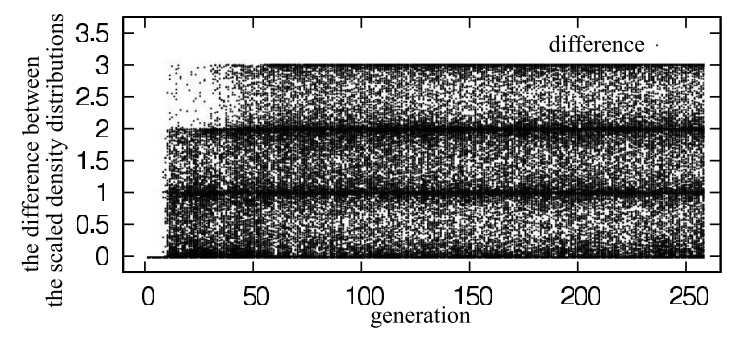

Fig. 4. The difference between the scaled density distributions.

As defined in Eq. 6, the fitness is the sum of the differences between the scaled density distributions. So as to grasp the main reason for the increase in fitness through the experiment, we plotted the all differences measured during the evaluation processes of all individuals at each generation in Fig. 4. After approximately the 30th generation, we see that the difference often became the maximum value 3.0 which was obtained when the difference between the mutually opposite distributions were measured. It clearly shows that the evolved CAs successfully exhibited the self-organizing behaviors that their global configurations drastically changed after the occurrences of external perturbations.

\section{B. Emergence of State Transition Cycles induced by External Perturbations}

In the previous section, we observed that the population successfully evolved, and individuals were expected to exhibit self-organizing behaviors induced by perturbations. Next, we analyze the behavior of the adaptive individuals in detail, and discuss their self-organizing properties.

Fig. 5 illustrates the transitions of several indices during an evaluation of a typical individual in the last generation of the same run as in Fig. 3. The string of genes is "020110010011110120010111010121012010000000000"2.

The lower graph shows the transitions of the elements of density distribution and the entropy ${ }^{3}$ during the evaluation. The above images also illustrate the configuration of cell states at the end of each period.

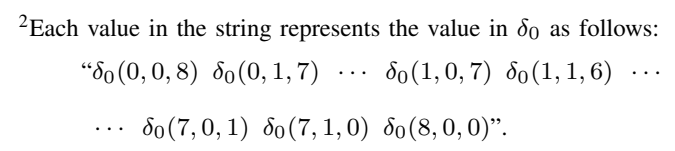

${ }^{3}$ The entropy of the global configuration $\mathbf{H}$ is defined by

$$
\begin{aligned}
\mathbf{H} & =\frac{1}{N \times N} \sum_{(i, j)} \in N \times N \\
H_{i, j} & =-\sum_{s=0}^{M-1} P_{i, j},
\end{aligned}
$$

where $H_{i, j}$ is the entropy of the cell $(i, j)$, and $P_{i, j}(s)$ is the probability of the occurrences of the cell state $s$ at $(i, j)$ during each period. 


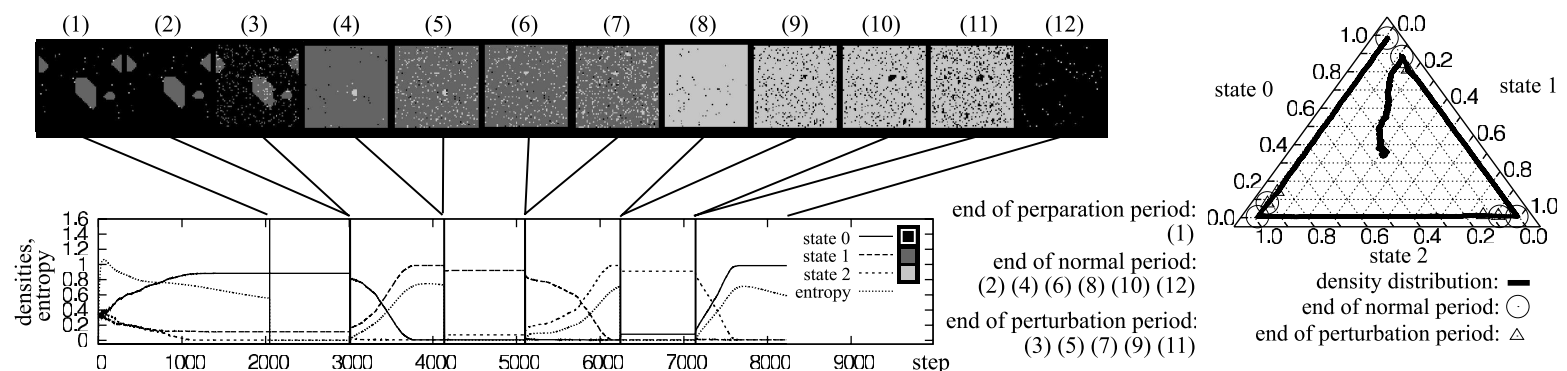

Fig. 5. The behavior of cellular automata (ascending cycle).

Fig. 6. The trajectory of density distribution (ascending cycle).

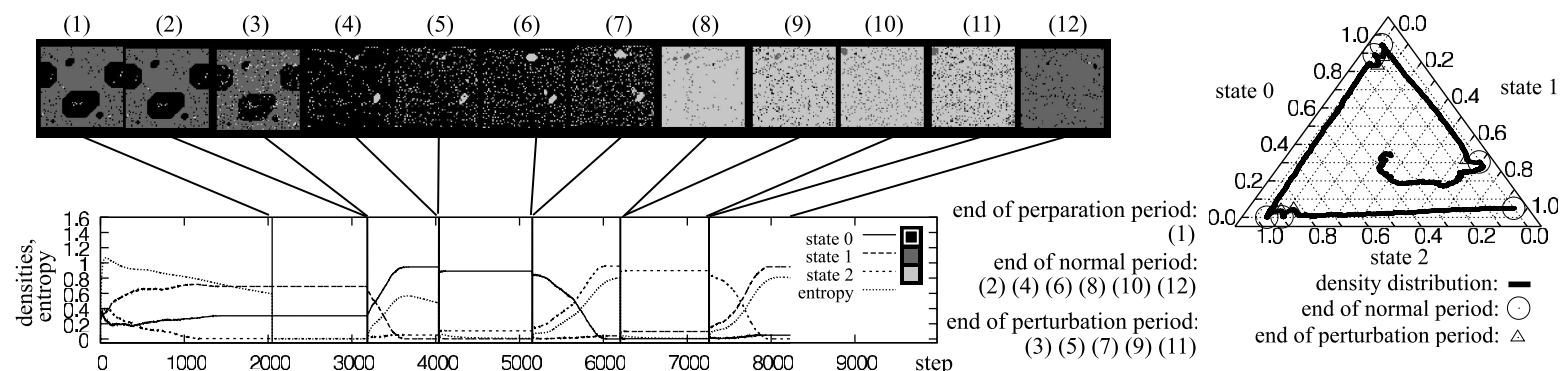

Fig. 7. The behavior of cellular automata (descending cycle).

Fig. 8. The trajectory of density distribution (descending cycle).

Through the preparation period, the configuration of the CA gradually changed from the random initial configuration to the stable one which was characterized by the decrease in the entropy. At the end of the preparation period, there were small clusters of the state 1 in a sea of the state 0 (Fig. 5 1). The configuration did not change through the first normal period (2).

Because the most of the cell states were 0 , the occurrences of the first perturbation period increased the density of the state 1 (3). Then, the clusters of 1 gradually expanded their size and finally occupied the whole configuration through the subsequent normal period (4).

In contrast, the effect of the second perturbation period was not strong. Although the density of the state 2 was increased (5), the global configuration did not change any further (6). However the effect of the third perturbation period (7) caused the significant change in the global configuration (8). The clusters of the state 2 appeared, expanded their size, and finally occupied the whole configuration. As explained, we observed similar changes in the dominant cell state (12) every two occurrences of perturbation periods (9 11). The detailed analyses on the effects of perturbations on the local interactions among cells showed that if the number of the subsequent dominant cell state exceeds a certain threshold by perturbations, it begins to increase during the subsequent normal period.

Fig. 6 is the trajectory of the density distribution during this evaluation. We see that the density distribution showed a triangle cycle on this space as a result of the emergent dynamics explained above. This cycle is ascending in that the value of the dominant cell state increases as the global configuration changes.

The global configuration of the CA in Fig. 5 and 6 showed cyclic transitions between 6 different configurations occupied by almost one cell state (i. e. Fig. 5 - 4) or two cell states ( $i$. $e$. a number of state 2 in a sea of state $1,(6))$. Because the scaling function increases the small density of the cell state, the actual differences in these scaled density distributions become large, and as a result, the differences between the several pairs (i.e. (6) and (12)) become the highest. Also, in each normal period, the global configuration completely converges before the end of the period as shown in Fig. 5. Thus, we can say that the cyclic behavior emerged through the course of evolution because of these adaptive and stable properties.

On the other hand, we also observed another interesting rule at the last generation in other runs. The string of genes is " $112211000100211001212112222120222200000000000 "$ ". Its typical behavior is illustrated in Fig. 7 and 8. As we can see from this figure, the density distribution exhibited a reverse (descending) cycle in comparison with the previous one. This is an unexpected phenomenon because the global configuration changes in a descending order despite that perturbations increments the values of cell states. The detailed analyses also showed that the perturbations worked like a catalyst in that the perturbed cells can decrement the values of cell states in neighbors. For example, if the configuration consists of a sea of the state 0 , the perturbed cells of 1 can change their neighboring cell states of 0 to 2 .

Among the successful runs, the rules of CA that exhibited 
Transition probability between configurations.

\begin{tabular}{|r|ccccccc|}
\hline & $\{0\}$ & $\{0,1\}$ & $\{1\}$ & $\{1,2\}$ & $\{2\}$ & $\{2,0\}$ & $\{0,1,2\}$ \\
\hline$\{0\}$ & 0.137 & 0.687 & 0.176 & 0.000 & 0.000 & 0.000 & 0.000 \\
$\{0,1\}$ & 0.000 & 0.016 & 0.959 & 0.024 & 0.000 & 0.000 & 0.000 \\
$\{1\}$ & 0.000 & 0.000 & 0.147 & 0.668 & 0.185 & 0.000 & 0.000 \\
$\{1,2\}$ & 0.000 & 0.000 & 0.000 & 0.029 & 0.955 & 0.016 & 0.000 \\
$\{2\}$, & 0.185 & 0.000 & 0.000 & 0.000 & 0.152 & 0.663 & 0.000 \\
$\{2,0\}$ & 0.979 & 0.013 & 0.000 & 0.000 & 0.000 & 0.009 & 0.000 \\
$\{0,1,2\}$ & 0.000 & 0.313 & 0.000 & 0.313 & 0.000 & 0.188 & 0.188 \\
\hline
\end{tabular}

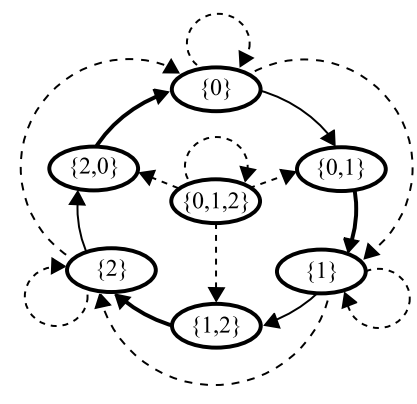

Fig. 9. The transition table and diagram for global configuration (ascending cycle).

Transition probability between configurations.

\begin{tabular}{|r|ccccccc|}
\hline & $\{0\}$ & $\{0,1\}$ & $\{1\}$ & $\{1,2\}$ & $\{2\}$ & $\{2,0\}$ & $\{0,1,2\}$ \\
\hline$\{0\}$ & 0.000 & 0.000 & 0.000 & 0.000 & 0.038 & 0.962 & 0.000 \\
$\{0,1\}$ & 0.721 & 0.208 & 0.000 & 0.000 & 0.000 & 0.071 & 0.000 \\
$\{1\}$ & 0.048 & 0.952 & 0.000 & 0.000 & 0.000 & 0.000 & 0.000 \\
$\{1,2\}$ & 0.000 & 0.059 & 0.648 & 0.293 & 0.000 & 0.000 & 0.000 \\
$\{2\}$ & 0.000 & 0.000 & 0.017 & 0.983 & 0.000 & 0.000 & 0.000 \\
$\{2,0\}$ & 0.000 & 0.000 & 0.000 & 0.086 & 0.676 & 0.239 & 0.000 \\
$\{0,1,2\}$ & 0.000 & 0.333 & 0.000 & 0.111 & 0.000 & 0.111 & 0.444 \\
\hline
\end{tabular}

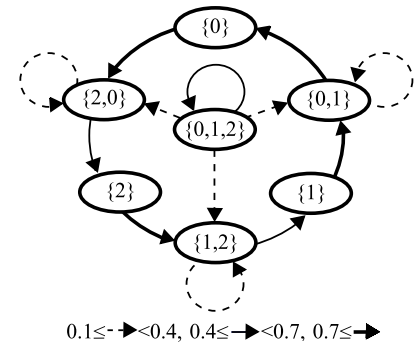

Fig. 10. The transition table and diagram for global configuration (descending cycle).

the ascending cycles were clearly observed in 5 runs, and those exhibited the descending cycles were obtained in 3 runs. We can say that there were two opposite solutions to solve the task, but both rules cyclically changed the global configuration of CAs by using their self-organizing properties that a drastic change in its global state occurs every if the number of the subsequent dominant cells goes beyond a certain threshold.

\section{Stability of Emerged Cycles}

We obtained the emergent behaviors of CAs with cyclic trajectories of the density distribution triggered by perturbations. However, it needs more consideration to discuss the stability of the cyclic behaviors of CAs during their long-term transitions because the number of the perturbation periods in each evaluation process was merely 5. Consequently, we conducted additional evaluations on the two typical rules explained in the previous section, in which the settings of the parameters were the same as the previous experiments except for the number of perturbation periods $D=17$.

In order to understand the stability of transitions between configurations, we calculated the transition probability between configurations at the steps when the global configurations were used for fitness evaluation $(a(0), a(1), \ldots, a(D)$ in Fig. 1). Specifically, all global configurations were divided into into $2^{3}=8$ classes depending on whether each cell state exist or not on the global configuration. The existence of a cell state on the global configuration was decided by whether the density of the cell state can be increased by the scaling function (Eq. (4)) or not ${ }^{4}$. For example, if a density distribution is $\{0.80,0.15,0.05\}$ and the scaled density distribution is $\{1.00,0.99,0.01\}$, then it is regarded that the cell state 0 and 1 exist on the configuration. Then, we measured the transition probabilities between the classes during the evaluation process.

The table in Fig. 9 displays the average transition probabilities between configurations over 100 evaluations of the individual which showed the ascending cycle in the previous experiment. Each set of cell states in row and column is the set of existent cell states in the corresponding class, and each value is the specific transition probability from the column to the row class. The transition diagram on the right also visualizes the same distribution probabilities, in which the line types of arrows correspond to different ranges of the value. As shown from the table and diagram, all the transition probabilities corresponding to the ascending cycle $\{0\} \rightarrow\{0,1\} \rightarrow\{1\} \rightarrow\{1,2\} \rightarrow\{2\} \rightarrow\{2,0\} \rightarrow \ldots$, were greater than 0.65 . As above, the ascending cycle with 6 different configurations is stable through the long-term evaluation.

The table in Fig. 10 displays the transition probability between configurations of the individual which showed the descending cycle in the previous experiments. The transition probabilities corresponding to the descending cycle $\{0\} \rightarrow$ $\{2,0\} \rightarrow\{2\} \rightarrow\{1,2\} \rightarrow\{1\} \rightarrow\{0,1\} \rightarrow \ldots$, were grater than 0.65 approximately, which are similar to the transition probabilities of the ascending cycle. The transition

\footnotetext{
${ }^{4}$ Actually, we defined that the density can be increased if the density is larger than the $\mathrm{x}$-value (approximately 0.075) at the intersection of $y=$ $S F_{0.1,100}(x)$ with $y=x$ around $\theta=0.1$.
} 
diagram clearly shows that the cycle is also stable while it is reversed compared to the previous one.

\section{CONCLUSions}

We have investigated emergent properties of CAs induced by external perturbations. We introduced the transitivity into an extended version of outer-totalistic rules of CAs, and adopted the scaled density distribution of cell states as the global configuration, expecting emergences of the selforganizing behaviors and the reduction of the search space for a GA. We assumed a minimal task in which a CA has to change its global state every perturbation, and then searched the rules for CAs which can solve the task by using a GA. We obtained the rules for the CA in which the global configuration cyclically transited among different stable configurations, and these stable configurations composed of not only homogeneous but also heterogeneous cell states. As a result, the number of stable configurations became twice as that of possible cell states. These interesting results were obtained only when we introduced the transitivity (Eq. (8)) into the rule of CAs. It should be emphasized that we found both ascending and descending cycles of global configurations even though a perturbation always increments the value of a cell state. Detailed analyses showed that the ascending cycle was due to the self-organizing feature that a drastic change in its global state occurs every when the accumulation of the perturbed cells goes beyond a certain threshold. Also, the descending cycle was due to the catalytic effect of perturbed cells which change cell states in their neighborhood into the dominant cell state in the subsequent stable configuration.

Mamei et al. argued about applications of self-organizing features in the dissipative CAs for controlling decentralized pervasive systems[6]. In our model, we can regard the external perturbations as the signals for controlling the global behavior of decentralized systems. Our results imply that we can dynamically control the global behaviors of decentralized systems by changing several states of randomly selected components only.

Future work includes investigating our model with different several parameters, obtaining more complex behaviors induced by different kinds of perturbations, such as conditional branches of the behavior trajectories based on the kinds of perturbations, and formulating in which a CA and an external environment mutually interact.

\section{ACKNOWLEDGMENT}

This work was supported in part by a Grant-in-Aid for 21st Century COE "Frontiers of Computational Science".

\section{REFERENCES}

[1] S. Wolfram, A New Kind of Science, Wolfram Media Inc. 2002.

[2] T. E. Ingerson and R. L. Buvel, "Structure in Asynchronous Cellular Automata," Physica D, vol. 10, pp. 59-68, Jan. 1984.

[3] S. Ninagawa, M. Yoneda and S. Hirose, "Cellular Automata in Dissipative Boundary Conditions (in Japanese)," Transactions of Information Processing Society of Japan, vol. 38, no. 4, pp. 927-930, Apr. 1997.
[4] C. Marr and M. T. Hütt, "Similar Impact of Topological and Dynamic Noise on Complex Patterns," Physics Letters A, vol. 349, pp. 302-305, Jan. 2006.

[5] A. Roli and F. Zambonelli, "Emergence of Macro Spatial Structures in Dissipative Cellular Automata," Proc. the 5th International Conference on Cellular Automata for Research and Industry (ACRI2002), pp. 144155, Oct. 2002.

[6] M. Mamei, A. Roli and F. Zambonelli, "Emergence and Control of Macro-Spatial Structures in Perturbed Cellular Automata, and Implications for Pervasive Computing Systems, "IEEE Trans. Systems, Man, and Cybernetics, Part A: Systems and Humans, vol. 35, vo. 3, pp. 337348, May 2005.

[7] M. Mitchell, J. P. Crutchfield and P. T. Hraber, "Evolving Cellular Automata to Perform Computations: Mechanisms and Impediments," Physica D, vol. 75, pp. 361-391, Aug. 1994.

[8] L. M. Rocha, "Evolving Memory: Logical Tasks for Cellular Automata," Proc. the 9th International Conference on the Simulation and Synthesis of Living Systems (ALIFE9), pp. 256-261, Sep. 2004.

[9] S. Ninagawa, "Evolving Cellular Automata by 1/f Noise," Proc. the 8th European Conference on Artificial Life (ECAL2005), pp. 453-460, Sep. 2005. 